\title{
O IMPACTO DA TECNOLOGIA DE INFORMAÇÃO NA ESTRATÉGIA DOS BANCOS
}

\author{
Antonio Carlos Gastaud Maçada \\ Mestre em Administração pela UFRGS, Doutor em Administração pela UFRGS \\ e Professor Adjunto do DCEAC da FURG. \\ E-mail: acgmacada@adm.ufrgs.br \\ João Luiz Becker \\ Mestre em Matemática Aplicada pelo IMPA, Ph.D. em Administração pela UCLA \\ e Professor Titular da EA da UFRGS. \\ E-mail: jlbecker@adm.ufrgs.br
}

\section{RESUMO}

O potencial da Tecnologia de Informação (TI) em capacitar e sustentar estratégias e sucesso organizacional está centrado na capacidade de seus dirigentes perceberem o valor e os impactos dos investimentos em TI nos negócios. Neste artigo, analisam-se as percepções dos executivos de bancos brasileiros e norte-americanos

quanto aos impactos da TI nas variáveis estratégicas organizacionais. O estudo revela semelhança de percepção entre executivos brasileiros e norte-americanos e também entre executivos das áreas de TI e de outras áreas. Quatro variáveis afetadas pela TI são os principais determinantes da competitividade na indústria bancária: requisitos de governos e países; clientes; estrutura de custos e capacidade; produtos e serviços.

\section{ABSTRACT}

The Information Technology (IT) potential in supporting strategies and the organizational success is based on executives' ability in perceiving the value and effects of IT investments in businesses. In this article, Brazilian and North-American banking executives perceptions are analyzed with regard to IT effects in the strategic organizational variables. The study points out the similarity of perception among Brazilian and North-American executives as well as among executives of IT areas and other areas. Four variables affected by IT are the main determinants of competitiveness in the banking industry: governments and countries' requirements; clients; cost structure and capacity; products and services.

\section{PALAVRAS-CHAVE}

TI, investimentos em TI, impacto da TI, bancos, competitividade.

\section{KEY WORDS}

IT, IT investment, IT impact, banking, competitiveness. 


\section{INTRODUÇÃO}

A capacidade dos executivos para guiar e influenciar ações relacionadas com gerenciamento e uso da Tecnologia de Informação (TI) nas estratégias dos negócios tem sido reconhecida como competência crítica nas organizações (Sambamurthy e Zmud, 1992, 1994). Não é por acaso que inúmeros trabalhos acadêmicos têm-se dedicado a analisar essa temática (Baets, 1996; Rota, 1998; Khiaonarong e Liebenau, 1999). Armstrong e Sambamurthy (1999), entretanto, salientam que, em muitos artigos, na maioria prescritivos, pesquisas empíricas e estudos de caso apresentam análises fragmentadas. Por exemplo, somente são avaliadas a influência e as diferentes percepções com relação a habilidades e conhecimentos dos executivos da área de tecnologia e da alta administração. Em contraste, Rockart et al. (1996) e O'Brien (1999) destacam que o uso e o gerenciamento da TI são responsabilidades de todos os executivos. O envolvimento do total de executivos (de TI e administração) é a melhor maneira de assegurar que as estratégias de TI estejam alinhadas às estratégias de negócios e que os investimentos estejam direcionados de forma a atender às necessidades das organizações (Kempis et al., 1999).

\section{É IMPORTANTE AS ORGANIZAÇÕES}

\section{UNIREM HABILIDADES GENERALISTAS}

\section{VITAIS COM AS DOS ESPECIALISTAS}

\section{EM TI PARA FORMAR UMA}

VISÃO ESTRATÉGICA ÚNICA

\section{DE TI E DE NEGÓCIOS.}

É importante que as organizações unam habilidades generalistas vitais com as dos especialistas em TI com a meta de formar uma visão estratégica única de TI e de negócios. Não é possível estruturar uma organização eficiente apenas com a estratégia de informação e a aplicação dos recursos de TI. É necessário também combinar a estratégia de negócios e recursos humanos (Morris et al., 1998).

Segundo Broadbent e Weill (1993), os bancos são as organizações que mais operam em um ambiente estratégico e competitivo, em que a atenção para o alinhamento dos negócios e das estratégias de informação deve ser o principal foco no esforço organizacional. Entretanto, apesar de a indústria bancária ter sido a primeira a desenvolver e utilizar a tecnologia orientada para os negócios, os retornos financeiros ainda não apresentaram resultados satisfatórios (Strassman, 1997; Lucas Jr., 1999). Kempis et al. (1999) ressaltam que a avaliação de investimentos em TI não pode restringirse a uma simples análise financeira, devendo incorporar outros métodos de análise. Uma das formas propostas por diversos autores (Mahmood e Soon, 1991; Palvia, 1997; Maçada et al., 2000) consiste na análise dos impactos desses investimentos em variáveis estratégicas organizacionais.

Dedrick e Kraemer (1995) afirmam que poucas pesquisas em Sistemas de Informação (SI) comparam dados entre países no contexto organizacional. Alguns estudos analisam diferentes países comparando políticas nacionais de TI e desenvolvimento econômico (Tam, 1998). Com base em recente revisão na literatura na área de SI, constatou-se que nenhum estudo examina e compara os dados brasileiros e norte-americanos no contexto organizacional. Pode-se especular que tal escassez de estudos comparativos deve-se à ausência de instrumentos validados de pesquisa em diferentes países.

$\mathrm{Na}$ medida em que os 30 maiores bancos norteamericanos possuem, ou estão estabelecendo, escritórios e filiais no Brasil (Warner, 1998), faz sentido identificar, analisar e comparar os impactos dos investimentos em TI nos bancos brasileiros e norte-americanos. Especificamente, este estudo focaliza duas questõeschave: a) Qual a percepção dos executivos de bancos no Brasil e nos Estados Unidos sobre os impactos da TI nas variáveis estratégicas organizacionais? São elas distintas? b) Qual a relação entre os impactos da TI sobre as variáveis estratégicas organizacionais e o impacto da TI sobre a competitividade da organização? Os dados para responder a essas questões foram coletados por meio de uma pesquisa realizada em bancos no Brasil, filiados à Federação Brasileira das Associações de Bancos (Febraban), e em bancos norteamericanos, filiados à American Banks Association (ABA). Os participantes são executivos da área de tecnologia e das diversas áreas administrativas.

\section{TI E BANCOS NORTE- AMERICANOS E BRASILEIROS}

De acordo com Morisi (1996), a introdução da TI nos bancos norte-americanos aconteceu em 1950, quando o primeiro computador foi desenvolvido e montado para o Bank of America. Segundo Pires (1996), uma das utilizações mais antigas de automação bancária no Brasil foi a implantação de sistemas de controle em 1956. Inicialmente, os computadores foram utilizados para processar e controlar os cheques emitidos pelos clientes. Nos anos 70, surgiram as primeiras máquinas ATM (Automated Teller Machine) nos Estados Unidos, com o objetivo de oferecer produtos e serviços, atrair 
consumidores e reduzir custos. Essa novidade tecnológica chegou ao Brasil somente em 1983.

Rapidamente, as ATMs passaram a ser utilizadas como um dos principais instrumentos de competição entre os bancos (Peffers e Dos Santos, 1996). A tecnologia das ATMs proporcionou produtos e serviços para os clientes 24 horas por dia, nos sete dias da semana, em qualquer ponto geográfico. A tecnologia de telefonia (call centers) tem sido uma alternativa de investimento bastante atrativa para os bancos, com custo médio por transação correspondendo, nos Estados Unidos, a US\$ 0,54 (Galbreath, 1998). A Internet incorpora-se ao conjunto de tecnologias bancárias na metade da década de 90, transformando-se em um canal estratégico, aumentando significativamente as opções de investimentos em TI.

Grandes bancos norte-americanos como Citicorp, Chase Manhattan e Bank of America têm realizado vultosos investimentos em TI - US\$1,9 bilhão, US\$ 1,8 bilhão e US\$ 1,2 bilhão respectivamente em 1996, segundo Schifrin (1997). Cada um desses valores é comparável ao montante investido pela indústria bancária de alguns países em desenvolvimento, como o Brasil, cujo investimento de toda a indústria bancária totalizou R \$ 1,5 bilhão em 1996 (Febraban, 1997). Apesar da disparidade de cifras, percebe-se claramente que os bancos de ambos os países têm usado a TI como principal instrumento para implementação de suas estratégias.

\section{MODELO CONCEITUAL DA PESQUISA}

A teoria da estratégia e competitividade liderada por Porter (1980) contribuiu para o desenvolvimento de vários estudos na literatura de SI. Os artigos que utilizam essa teoria procuram identificar a capacidade da TI em alterar a maneira de as empresas operarem, em transformar a cadeia de valor e em apoiar a implementação de estratégias (Mahmood e Soon, 1991). A base teórica da pesquisa está fundamentada em dois modelos distintos, desenvolvidos por Mahmood e Soon (1991) e por Palvia (1997). O primeiro mede o impacto da TI nas variáveis estratégicas organizacionais em contexto nacional. $\mathrm{O}$ segundo, usando a mesma estrutura formal do primeiro, avalia o impacto da TI em contexto global. Esta pesquisa combina os dois modelos, já que as unidades de análise - os bancos - inserem-se em ambientes competitivos tanto no país-sede como internacionalmente.

O instrumento utilizado foi desenvolvido e validado no Brasil (Maçada e Becker, 1998), sendo, posteriormente, validado nos Estados Unidos (Maçada et $a l .$, 2000). Em sua versão final, o instrumento totaliza 12 variáveis operacionalizadas por 35 itens, utilizando escalas Likert de 5 pontos. O coeficiente de fidedignidade (alpha de Cronbach) do instrumento é de 0,90 para o Brasil e 0,95 para os Estados Unidos. Os coeficientes das variáveis que o compõem situam-se entre 0,71 e 0,95 (Brasil) e entre 0,61 e 0,83 (Estados Unidos). A qualidade do instrumento foi confirmada utilizando-se a abordagem de Modelagem de Equações Estruturais, mais especificamente o modelo fatorial confirmatório (modelo de mensuração), apoiado no software estatístico LISREL (Linear Structural Relationships) (Jöreskog e Sörbom, 1996). O Índice de Qualidade do Ajustamento (Goodness of Fit Index (GFI)) obtido foi 0,90, considerado adequado (Segars e Grover, 1993; Hartwick e Barki, 1994).

A Figura 1 apresenta o modelo de pesquisa resultante do processo de refinamento do instrumento utilizado, salientando as 12 variáveis operacionalizadas ${ }^{1}$, que fundamentam a percepção estratégica da TI e dos negócios na ótica dos executivos de bancos. Das 12

Figura 1 - Modelo conceitual

\begin{tabular}{|c|c|c|}
\hline & Variáveis estratégicas organizacionais & \\
\hline $\begin{array}{l}\text { Percepção } \\
\text { estratégica } \\
\text { da TI e dos } \\
\text { negócios }\end{array}$ & $\begin{array}{l}\text { - Tomadores de Recursos Financeiros (TRF) } \\
\text { - Fornecedores de Recursos Financeiros (FRF) } \\
\text { - Coleta e Troca de Informações (CTI) } \\
\text { - Produtos e Serviços (PeS) } \\
\text { - Estrutura de Custos e Capacidade (ECC) } \\
\text { - Eficiência Organizacional Interna (EOI) } \\
\text { - Eficiência Interorganizacional (EIN) } \\
\text { - Preços (PRE) } \\
\text { - Internacionalização (INT) } \\
\text { - Requisitos de Governos e Países (RGP) } \\
\text { - Coordenação Interorganizacional (CIN) }\end{array}$ & $\begin{array}{l}\text { M } \\
\mathbf{P} \\
\mathbf{E} \\
\mathbf{T} \\
\mathbf{I} \\
\mathbf{T} \\
\mathrm{I} \\
\mathrm{V} \\
\mathrm{I} \\
\mathrm{D} \\
\mathrm{A} \\
\mathrm{D} \\
\mathrm{E} \\
\text { (COM) }\end{array}$ \\
\hline
\end{tabular}


variáveis operacionalizadas, 11 focalizam determinados aspectos estratégicos da organização bancária e, possivelmente, afetam a percepção global de competitividade.

A amostra do estudo compreendeu 141 executivos de 68 bancos brasileiros (de um total de 125 filiados à Febraban) e 84 executivos de 52 bancos norte-americanos (do total de 300 maiores bancos filiados à $\mathrm{ABA}$ ). Os dados foram coletados no período de maio a outubro de 1998, no Brasil, e de fevereiro a junho de 1999, nos Estados Unidos. Os bancos que compõem a amostra brasileira representam $79 \%$ do patrimônio líquido total dos bancos brasileiros associados à Febraban. Todos os 50 maiores bancos brasileiros, segundo o ranking da revista Exame - Melhores e Maiores (Militello, 1998), responderam ao questionário. Os bancos que integram a amostra norte-americana representam $64 \%$ do patrimônio líquido total dos bancos norteamericanos. Os 40 maiores bancos norte-americanos, de acordo com o ranking divulgado pela revista The Banker (Blanden, 1998), responderam ao questionário.

\section{ANÁLISE DOS RESULTADOS}

Nesta seção, são apresentados os resultados obtidos. Ela divide-se em duas subseções, visando responder às duas questões-chave da pesquisa.

\section{Percepção dos executivos}

Para responder à primeira questão-chave do estudo, compararam-se as percepções dos executivos brasileiros e norte-americanos, segmentados por função exercida nas organizações - executivos de TI (EXETI) e de outras áreas funcionais (EXEADM) - quanto aos impactos da TI nas variáveis estratégicas dos bancos, por meio das 12 variáveis do instrumento. Mais especificamente, compararam-se as médias dessas variáveis entre os diversos grupos de executivos usandose a técnica de análise de variância multivariada (MANOVA) com dois fatores, disponível no aplicativo SPSS for Windows. A técnica de análise de variância multivariada é semelhante à análise de variância univariada. Ambas demonstram a existência de diferenças significativas entre os países (BRA x EUA) e as funções (EXETI x EXEADM); a análise univariada (ANOVA) trata cada uma das variáveis em separado, enquanto a análise multivariada (MANOVA) considera todas simultaneamente (Ruiz, 1999). A vantagem da técnica multivariada é seu grande poder de síntese. As Tabelas 1 e 2 apresentam as estatísticas descritivas das variáveis investigadas para cada combinação dos diferentes níveis das variáveis independentes. A Tabela 3 apresenta os resultados dos testes realizados.

As quatro estatísticas de contraste utilizadas para a variável "país" coincidem na rejeição da hipótese nula

\section{Tabela 1 - Estatísticas descritivas}

\begin{tabular}{|c|c|c|c|c|c|}
\hline Variável & País & Executivos & Média(*) & Desvio-padrão & $\mathbf{N}$ \\
\hline \multirow{3}{*}{ TRF } & Brasil & $\begin{array}{l}\text { EXETI } \\
\text { EXEADM } \\
\text { Total }\end{array}$ & $\begin{array}{l}4,4583 \\
4,3203 \\
4,3830\end{array}$ & $\begin{array}{l}0,5057 \\
0,5734 \\
0,5462\end{array}$ & $\begin{array}{r}64 \\
77 \\
141\end{array}$ \\
\hline & EUA & $\begin{array}{l}\text { EXETI } \\
\text { EXEADM } \\
\text { Total }\end{array}$ & $\begin{array}{l}4,0000 \\
3,8815 \\
3,9365\end{array}$ & $\begin{array}{l}0,7454 \\
0,7218 \\
0,7308\end{array}$ & $\begin{array}{l}39 \\
45 \\
84\end{array}$ \\
\hline & Total & $\begin{array}{l}\text { EXETI } \\
\text { EXEADM } \\
\text { Total }\end{array}$ & $\begin{array}{l}4,2848 \\
4,1585 \\
4,2163\end{array}$ & $\begin{array}{l}0,6441 \\
0,6642 \\
0,6566\end{array}$ & $\begin{array}{l}103 \\
122 \\
225\end{array}$ \\
\hline \multirow{3}{*}{ COM } & Brasil & $\begin{array}{l}\text { EXETI } \\
\text { EXEADM } \\
\text { Total }\end{array}$ & $\begin{array}{l}4,6094 \\
4,4481 \\
4,5213\end{array}$ & $\begin{array}{l}0,4836 \\
0,5769 \\
0,5407\end{array}$ & $\begin{array}{r}64 \\
77 \\
141\end{array}$ \\
\hline & EUA & $\begin{array}{l}\text { EXETI } \\
\text { EXEADM } \\
\text { Total }\end{array}$ & $\begin{array}{l}4,2051 \\
3,9889 \\
4,0893\end{array}$ & $\begin{array}{l}0,8006 \\
0,7941 \\
0,7997\end{array}$ & $\begin{array}{l}39 \\
45 \\
84\end{array}$ \\
\hline & Total & $\begin{array}{l}\text { EXETI } \\
\text { EXEADM } \\
\text { Total }\end{array}$ & $\begin{array}{l}4,4563 \\
4,2787 \\
4,3600\end{array}$ & $\begin{array}{l}0,6497 \\
0,6984 \\
0,6809\end{array}$ & $\begin{array}{l}103 \\
122 \\
225\end{array}$ \\
\hline
\end{tabular}


(continuação)

\begin{tabular}{|c|c|c|c|c|c|}
\hline Variável & País & Executivos & Média(*) & Desvio-padrão & $\mathbf{N}$ \\
\hline \multirow{9}{*}{ FRF } & \multirow{3}{*}{ Brasil } & EXETI & 4,2083 & 0,8389 & 64 \\
\hline & & EXEADM & 4,1082 & 0,7900 & 77 \\
\hline & & Total & 4,1537 & 0,8112 & 141 \\
\hline & \multirow{3}{*}{ EUA } & EXETI & 3,9915 & 0,8532 & 39 \\
\hline & & EXEADM & 3,7185 & 0,8070 & 45 \\
\hline & & Total & 3,8452 & 0,8351 & 84 \\
\hline & \multirow{3}{*}{ Total } & EXETI & 4,1262 & 0,8468 & 103 \\
\hline & & EXEADM & 3,9645 & 0,8152 & 122 \\
\hline & & Total & 4,0385 & 0,8318 & 225 \\
\hline \multirow{9}{*}{ CTI } & \multirow{3}{*}{ Brasil } & EXETI & 4,1172 & 0,7804 & 64 \\
\hline & & EXEADM & 3,9026 & 0,7949 & 77 \\
\hline & & Total & 4,0000 & 0,7928 & 141 \\
\hline & \multirow{3}{*}{ EUA } & EXETI & 3,9359 & 0,7962 & 39 \\
\hline & & EXEADM & 3,6444 & 0,6877 & 45 \\
\hline & & Total & 3,7798 & 0,7499 & 84 \\
\hline & \multirow{3}{*}{ Total } & EXETI & 4,0485 & 0,7875 & 103 \\
\hline & & EXEADM & 3,8074 & 0,7645 & 122 \\
\hline & & Total & 3,9178 & 0,7827 & 225 \\
\hline \multirow{9}{*}{ Pes } & \multirow{3}{*}{ Brasil } & EXETI & 4,4427 & 0,6231 & 64 \\
\hline & & EXEADM & 4,2641 & 0,6406 & 77 \\
\hline & & Total & 4,3452 & 0,6367 & 141 \\
\hline & \multirow{3}{*}{ EUA } & EXETI & 4,0940 & 0,7212 & 39 \\
\hline & & EXEADM & 4,0667 & 0,6341 & 45 \\
\hline & & Total & 4,0794 & 0,6719 & 84 \\
\hline & \multirow{3}{*}{ Total } & EXETI & 4,3107 & 0,6800 & 103 \\
\hline & & EXEADM & 4,1913 & 0,6427 & 122 \\
\hline & & Total & 4,2459 & 0,6613 & 225 \\
\hline \multirow{9}{*}{ ECC } & \multirow{3}{*}{ Brasil } & EXETI & 3,9180 & 0,6269 & 64 \\
\hline & & EXEADM & 3,8799 & 0,6534 & 77 \\
\hline & & Total & 3,8972 & 0,6395 & 141 \\
\hline & \multirow{3}{*}{ EUA } & EXETI & 3,9231 & 0,5853 & 39 \\
\hline & & EXEADM & 3,6778 & 0,5681 & 45 \\
\hline & & Total & 3,7917 & 0,5858 & 84 \\
\hline & \multirow{3}{*}{ Total } & EXETI & 3,9199 & 0,6086 & 103 \\
\hline & & EXEADM & 3,8053 & 0,6286 & 122 \\
\hline & & Total & 3,8578 & 0,6208 & 225 \\
\hline \multirow{9}{*}{ EOI } & \multirow{3}{*}{ Brasil } & EXETI & 4,0344 & 0,6700 & 64 \\
\hline & & EXEADM & 4,0857 & 0,5753 & 77 \\
\hline & & Total & 4,0624 & 0,6184 & 141 \\
\hline & \multirow{3}{*}{ EUA } & EXETI & 3,9897 & 0,6034 & 39 \\
\hline & & EXEADM & 3,8711 & 0,5413 & 45 \\
\hline & & Total & 3,9262 & 0,5706 & 84 \\
\hline & \multirow{3}{*}{ Total } & EXETI & 4,0175 & 0,6430 & 103 \\
\hline & & EXEADM & 4,0066 & 0,5703 & 122 \\
\hline & & Total & 4,0116 & 0,6033 & 225 \\
\hline
\end{tabular}

(*) Escala Likert de 1 (pouco) a 5 (muito). 
Tabela 2 - Estatísticas descritivas

\begin{tabular}{|c|c|c|c|c|c|}
\hline Variável & País & Executivos & Média $^{(*)}$ & Desvio-padrão & $\mathbf{N}$ \\
\hline \multirow{9}{*}{ EIN } & \multirow{3}{*}{ Brasil } & EXETI & 4,3490 & 0,6835 & 64 \\
\hline & & EXEADM & 4,3030 & 0,6886 & 77 \\
\hline & & Total & 4,3239 & 0,6842 & 141 \\
\hline & \multirow{3}{*}{ EUA } & EXETI & 3,8803 & 0,6861 & 39 \\
\hline & & EXEADM & 3,7259 & 0,6901 & 45 \\
\hline & & Total & 3,7976 & 0,6884 & 84 \\
\hline & \multirow{3}{*}{ Total } & EXETI & 4,1715 & 0,7184 & 103 \\
\hline & & EXEADM & 4,0902 & 0,7411 & 122 \\
\hline & & Total & 4,1274 & 0,7303 & 225 \\
\hline \multirow{9}{*}{ PRE } & \multirow{3}{*}{ Brasil } & EXETI & 4,3594 & 0,7425 & 64 \\
\hline & & EXEADM & 4,2078 & 0,7795 & 77 \\
\hline & & Total & 4,2766 & 0,7640 & 141 \\
\hline & \multirow{3}{*}{ EUA } & EXETI & 3,8077 & 0,9432 & 39 \\
\hline & & EXEADM & 3,8667 & 0,7790 & 45 \\
\hline & & Total & 3,8393 & 0,8543 & 84 \\
\hline & \multirow{3}{*}{ Total } & EXETI & 4,1505 & 0,8627 & 103 \\
\hline & & EXEADM & 4,0820 & 0,7935 & 122 \\
\hline & & Total & 4,1133 & 0,8247 & 225 \\
\hline \multirow{9}{*}{ INT } & \multirow{3}{*}{ Brasil } & EXETI & 4,3958 & 0,7672 & 64 \\
\hline & & EXEADM & 4,2944 & 0,7132 & 77 \\
\hline & & Total & 4,3404 & 0,7373 & 141 \\
\hline & \multirow{3}{*}{ EUA } & EXETI & 3,8889 & 0,7228 & 39 \\
\hline & & EXEADM & 3,8889 & 0,7588 & 45 \\
\hline & & Total & 3,8889 & 0,7378 & 84 \\
\hline & \multirow{3}{*}{ Total } & EXETI & 4,2039 & 0,7869 & 103 \\
\hline & & EXEADM & 4,1448 & 0,7533 & 122 \\
\hline & & Total & 4,1719 & 0,7677 & 225 \\
\hline \multirow{9}{*}{ RGP } & \multirow{3}{*}{ Brasil } & EXETI & 4,5000 & 0,4283 & 64 \\
\hline & & EXEADM & 4,4848 & 0,4574 & 77 \\
\hline & & Total & 4,4917 & 0,4429 & 141 \\
\hline & \multirow{3}{*}{ EUA } & EXETI & 3,8803 & 0,8002 & 39 \\
\hline & & EXEADM & 3,7037 & 0,7760 & 45 \\
\hline & & Total & 3,7857 & 0,7875 & 84 \\
\hline & \multirow{3}{*}{ Total } & EXETI & 4,2654 & 0,6656 & 103 \\
\hline & & EXEADM & 4,1967 & 0,7026 & 122 \\
\hline & & Total & 4,2281 & 0,6852 & 225 \\
\hline \multirow{9}{*}{ CIN } & \multirow{3}{*}{ Brasil } & EXETI & 4,0781 & 0,6739 & 64 \\
\hline & & EXEADM & 4,0519 & 0,7547 & 77 \\
\hline & & Total & 4,0638 & 0,7168 & 141 \\
\hline & \multirow{3}{*}{ EUA } & EXETI & 3,7949 & 0,9012 & 39 \\
\hline & & EXEADM & 3,7222 & 0,8087 & 45 \\
\hline & & Total & 3,7560 & 0,8484 & 84 \\
\hline & \multirow{3}{*}{ Total } & EXETI & 3,9709 & 0,7759 & 103 \\
\hline & & EXEADM & 3,9303 & 0,7881 & 122 \\
\hline & & Total & 3,9489 & 0,7811 & 225 \\
\hline
\end{tabular}

(*) Escala Likert de 1 (pouco) a 5 (muito). 
de inexistência de diferenças, com um nível de significância elevado. Desta forma, pode-se afirmar, com segurança, que os executivos brasileiros e norteamericanos diferem quanto à percepção do impacto da TI nas variáveis estratégicas organizacionais nos bancos. Já para o caso da variável "executivos", o teste realizado não permite rejeitar a hipótese nula de inexistência de diferenças $(\alpha=0,447)$. Em outras palavras, as percepções dos executivos da área de tecnologia e das áreas administrativas quanto aos impactos da TI nas variáveis estratégicas organizacionais nos bancos são semelhantes. Tais resultados são auspiciosos, na medida em que boa parte da literatura sobre o uso efetivo de TI nas organizações ressalta a importância da interação e compartilhamento de visões entre seus altos executivos e os executivos da área de TI (Armstrong e Sambamurthy, 1999). Pode-se esperar, portanto, um uso efetivo da TI pelos bancos brasileiros e norte-americanos. Não é surpresa constatar que a indústria bancária é a que mais tem investido em TI ultimamente (Lucas Jr., 1999). Quanto a possíveis efeitos de interação entre as duas variáveis independentes, estes revelam-se não significativos $(\alpha=$ $0,854)$, não permitindo rejeitar a hipótese nula de inexistência do efeito interação. A interação entre país de origem e função exercida não gera diferenças na percepção dos efeitos da TI nas variáveis estratégicas organizacionais nos bancos.
Analisando os dados constantes nas Tabelas 1 e 2, observa-se que as médias de todas as variáveis investigadas são maiores para os executivos brasileiros do que para os norte-americanos. Em outros termos, os executivos brasileiros percebem os impactos da TI nas variáveis estratégicas organizacionais no ambiente organizacional (bancos) com maior intensidade do que os norte-americanos. Hofstede (1991) salienta haver diferenças culturais marcantes entre Brasil e Estados Unidos. Em particular, Zanela et al. (1998), focalizando o processo decisório, identificam diferenças culturais significativas entre executivos brasileiros e norte-americanos de diversas organizações. Não é surpresa, pois, que o presente estudo aponte diferenças de percepção do impacto da TI nos bancos entre os executivos dos dois países.

Inovações tecnológicas na indústria bancária, embora mais tardias no Brasil do que nos Estados Unidos, tendem a ser assimiladas com maior intensidade e penetração no Brasil do que nos Estados Unidos. Assim, por exemplo, o percentual de clientes on-line sobre o total de clientes do maior banco comercial do Brasil, o Bradesco, é de $23,4 \%$, superior, portanto, ao percentual de $15 \%$ do maior banco norte-americano de varejo, o Bank of America.

A disposição cultural para aceitação de TI no Brasil também é evidenciada pelo destaque internacional obtido pelos sistemas brasileiros de imposto de renda

Tabela 3 - Contrastes multivariados

\begin{tabular}{|l|c|c|c|c|c|}
\hline \multicolumn{1}{|c|}{ Efeito } & Valor & F & gl da hipótese & gl do erro & SIG \\
\hline Intercepto & & & & & \\
\hline Pillai's Trace & 0,990 & 1824,087 & 12 & 210 & 0,000 \\
Wilks' Lambda & 0,010 & 1824,087 & 12 & 210 & 0,000 \\
Hotelling's Trace & 104,234 & 1824,087 & 12 & 210 & 0,000 \\
Roy's Largest Root & 104,234 & 1824,087 & 12 & 210 & 0,000 \\
\hline PAí́S & & & & & \\
\hline Pillai's Trace & 0,315 & 8,049 & 12 & 210 & 0,000 \\
Wilks' Lambda & 0,685 & 8,049 & 12 & 210 & 0,000 \\
Hotelling's Trace & 0,460 & 8,049 & 12 & 210 & 0,000 \\
Roy's Largest Root & 0,460 & 8,049 & 12 & 210 & 0,000 \\
\hline EXECUTIVOS & & & & & \\
\hline Pillai's Trace & 0,054 & 1,003 & 12 & 210 & 0,447 \\
Wilks' Lambda & 0,946 & 1,003 & 12 & 210 & 0,447 \\
Hotelling's Trace & 0,057 & 1,003 & 12 & 210 & 0,447 \\
Roy's Largest Root & 0,057 & 1,003 & 12 & & 0,447 \\
\hline PAíS x EXECUTIVOS & & & & 210 & 0,854 \\
\hline Pillai's Trace & 0,032 & 0,584 & 12 & 210 & 0,854 \\
Wilks' Lambda & 0,968 & 0,584 & 12 & 210 & 0,854 \\
Hotelling's Trace & 0,033 & 0,584 & 12 & 210 & 0,854 \\
Roy's Largest Root & 0,033 & 0,584 & 12 & \\
\hline
\end{tabular}

Desenho: Intercepto + PAíS + EXECUTIVOS + PAÍS x EXECUTIVOS 
de pessoa física (Leite, 1998) e de urnas eletrônicas. Em virtude de a economia norte-americana e, em particular, de o mercado bancário serem mais estruturados e com maior tradição e organização, em contraste com o brasileiro, podem-se destacar os brasileiros como os que aceitam as inovações com mais benevolência.

\section{A AVALIAÇÃO DE INVESTIMENTOS}

\section{EM TI NÃO PODE RESTRINGIR-SE}

\section{A SIMPLES ANÁLISE FINANCEIRA, DEVENDO INCORPORAR OUTROS}

\section{MÉTODOS DE ANÁLISE.}

Por outro lado, o fato de o sistema bancário brasileiro estar atento à competição internacional no presente momento (Albright e Parker, 1998) - com a internacionalização de operações e a globalização dos mercados financeiros sendo alavancadas pelo uso da TI (Blanden, 1998) e, sobretudo, com controles sendo assumidos por congêneres estrangeiros (Warner, 1998) -, pode ser apontado como fator importante na consideração de possíveis causas da diferença de percepção entre os executivos dos dois países. É fato que os executivos de bancos brasileiros, segundo depoimento aos pesquisadores, percebem a TI como importante fator de agregação de valor com vistas a um possível processo de fusão ou incorporação por parte de bancos estrangeiros ou entre bancos nacionais.

Os impactos da TI são percebidos em diversas intensidades nas diferentes variáveis do estudo. Depreende-se das Tabelas 1 e 2 que os impactos da TI na variável "competitividade" são percebidos com maior intensidade do que nas demais variáveis estratégicas estudadas ( $m e ́ d i a$ Brasil = 4,52, média EUA = $4,09$, média geral $=4,36)^{2}$. Os dados não surpreendem, tendo em vista que a variável "competitividade" expressa genericamente o que a literatura de SI apresenta como principal fator para utilização da TI (Porter e Millar, 1985; Rockart et al., 1996; Callahan e Nemec Jr., 1999). As demais variáveis operacionalizadas, todas em certa medida ligadas à noção geral de competitividade, segmentam aspectos mais específicos ligados à operação dos bancos.

Identificam-se grupos de variáveis estratégicas ordenados pela intensidade dos impactos conseqüentes da utilização da TI, segundo a percepção dos executivos dos bancos pesquisados. Há um conjunto de variáveis que apresenta maior intensidade dos impactos advindos do uso da TI: "produtos e serviços" (média geral $=4,25$ ), "requisitos de governo e países" (média geral $=4,23$ ), "tomadores de recursos financeiros" (média geral $=4,22$ ), "internacionalização" (média geral $=4,17)$, "eficiência interorganizacional" (média geral $=4,13$ ) e "preços" (média geral $=4,11$ ), em contraste com as variáveis "fornecedores de recursos financeiros" (média geral = 4,04), "eficiência organizacional interna" (média geral = 4,01), "coordenação interorganizacional" (média geral $=3,95$ ), "coleta e troca de informações" (média geral $=3,92$ ) e "estrutura de custos e capacidade" (média geral = $3,86)$.

Os resultados refletem o atual contexto competitivo em que os bancos estão inseridos, com perspectivas de fusões e aquisições tanto internacionais como nacionais (Sijbrands e Eppink, 1994; Albright e Parker, 1998). Por outro lado, a TI, particularmente com máquinas ATM e Internet, tem possibilitado o desenvolvimento e distribuição de novos produtos e serviços diferenciados, conquistando os clientes (Devlin, 1995; Lucas Jr., 1999; Jordan e Katz, 1999).

\section{Relação entre os impactos da TI}

A segunda questão-chave da pesquisa relaciona-se à possível relação entre o impactos da TI sobre as variáveis estratégicas organizacionais específicas e o impacto da TI sobre a competitividade geral da organização. Procurando identificar tal relação, utilizou-se um modelo de regressão múltipla envolvendo as variáveis estudadas, com o constructo "competitividade" como variável dependente. Dois modelos foram testados, usando os dados das subamostras norte-americanas e brasileiras respectivamente. Para a amostra norteamericana, os resultados revelam a interação entre quatro variáveis estatisticamente significativas (ver Figura 2): "internacionalização"; "estrutura de custos e capacidade"; "tomadores de recursos financeiros"; e "fornecedores de recursos financeiros" $\left(\mathrm{r}=0,744, \mathrm{r}^{2}\right.$ ajustado $=0,531, \alpha=0,000$ ). As demais variáveis estudadas não apresentaram coeficientes significativamente diferentes de zero $(\mathrm{p}<0,05)$.

Na percepção dos executivos norte-americanos, os impactos da TI na competitividade dos bancos estão relacionados com os impactos da TI nas variáveis que representam a combinação de fatores de competição internacional ("internacionalização"), de clientes ("tomadores e fornecedores de recursos financeiros") e de aspectos administrativos organizacionais ("estrutura de custos e capacidade").

As variáveis selecionadas pelo modelo de regressão representam quatro dimensões fundamentais para a obtenção de competitividade na indústria bancária, o que encontra respaldo na literatura. Conforme Jordan e Katz (1999, p. 28), "as mudanças trazidas pela TI novos produtos e serviços, clientes mais sofisticados, 
mudanças na estrutura de custos e pressões competitivas ampliadas - transformaram a estrutura da indústria bancária”. Peffers e Tuunainen (1998) relatam o caso do sistema de informação interorganizacional denominado HSBC Hexagon, desenvolvido para o Banco HSBC. O sistema oferece aos clientes serviços bancários em qualquer lugar do mundo 24 horas por dia, 365 dias por ano. Os autores ressaltam que o sistema foi crucial ao gerar economias de escopo e de escala, elevando o valor dos produtos e serviços para os consumidores, aumentando a eficiência e a efetividade organizacionais e, conseqüentemente, proporcionando ao referido banco importantes vantagens competitivas. Recentemente, o Citigroup anunciou planos de lançamento de um portal oferecendo serviços de comércio eletrônico a seus mais de 100 milhões de consumidores em cem países. De acordo com executivos do Citigroup, a iniciativa representa uma "poderosa combinação de tecnologia de ponta, a franquia corporativa global do Citigroup, e nossos serviços financeiros 'sem fronteiras" (www.bloomberg.com, acessado em 28 de fevereiro de 2000). O portal ampliará a base de clientes, terá condições de oferecer novos serviços aos clientes já existentes, com economia de custos e aumento de eficiência. Segundo Galbreath (1998), a Internet tem sido utilizada pelos bancos como mecanismo de corte de custos e de geração de novas fontes de receita, visando agregar valor para os clientes.
O procedimento aplicado à subamostra brasileira não se revela tão nítido. O modelo resultante indica a relação estatisticamente significativa de apenas uma variável (ver Figura 3): "estrutura de custos e capacidade" $\left(r=0,174, r^{2}\right.$ ajustado $\left.=0,023, \alpha=0,039\right)$. As demais variáveis estudadas não apresentaram coeficientes significativamente diferentes de zero $(p<0,05)$.

Na percepção dos executivos brasileiros, os impactos da TI na competitividade dos bancos estão relacionados apenas com os impactos da TI na estrutura de custos e capacidade.

Seguindo a tendência mundial, bancos brasileiros têm investido volumes expressivos no desenvolvimento de sistemas baseados na Internet. O Bradesco, por exemplo, vem investindo $\mathrm{R} \$ 250$ milhões anualmente em TI, tendo usado pioneiramente, na indústria bancária, o conceito de portal - o primeiro no Brasil e o quinto no mundo (http://www.bradesco.com.br/corpor/historia/ origens.html, acessado em 21 de março de 2000). A percepção dos executivos brasileiros parece estar ainda atrelada a uma visão limitada de back-office, com o foco da tecnologia voltado para a transformação e substituição de atividades internas do banco, e não direcionado para a implementação de estratégias. Recente pesquisa realizada pela ITMedia (Companhias, 2001) revela que as empresas brasileiras, em geral, preocuparam-se até 2000 em investir apenas em infra-estrutura de TI. O estudo prevê que, a partir de 2001, os investimentos em TI sejam voltados à implementação de estratégias.

\section{Figura 2 - Modelo de regressão para os Estados Unidos}

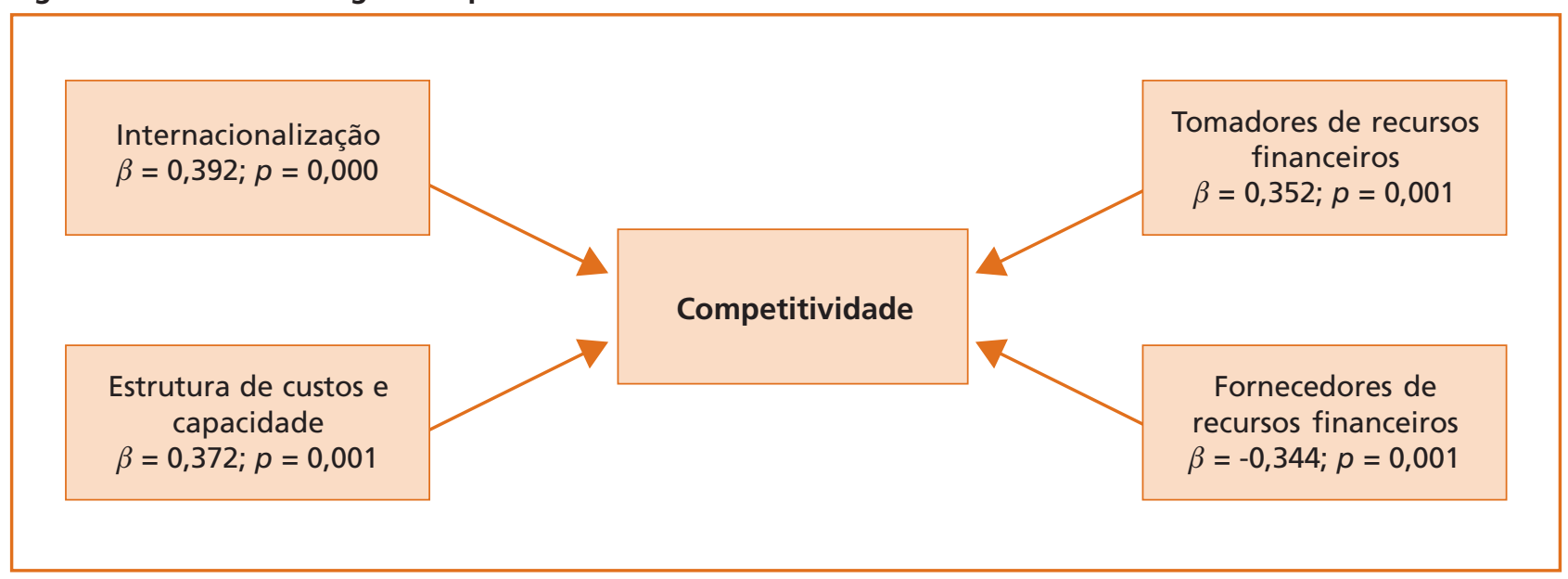

Figura 3 - Modelo de regressão para o Brasil






\section{CONCLUSÕES}

Nesta pesquisa, foram discutidas as percepções dos executivos da indústria bancária no Brasil e nos Estados Unidos quanto aos impactos da TI nas variáveis estratégicas organizacionais. Visando efetuar a análise, desenvolveu-se um instrumento capaz de avaliar

\section{As PERCEPÇÕES DOS EXECUTIVOS DA}

ÁREA DE TECNOLOGIA E DAS ÁREAS

ADMINISTRATIVAS QUANTO AOS

IMPACTOS DA TI NAS VARIÁVEIS

ESTRATÉGICAS ORGANIZACIONAIS

\section{NOS BANCOS SÃO SEMELHANTES.}

os impactos da TI em variáveis estratégicas organizacionais, conforme percepção dos executivos de todas as áreas estratégicas da organização. $\mathrm{O}$ instrumento disponibiliza medidas em 12 variáveis, operacionalizadas em 35 itens.

As conclusões fundamentais do estudo expõem a diferença de percepção entre executivos brasileiros e norte-americanos e a semelhança de percepção entre executivos das áreas de TI e de outras áreas. Executivos bancários brasileiros e norte-americanos percebem diferentemente os impactos da TI nas variáveis estratégicas. Executivos ligados à TI e demais executivos, independentemente do país, não diferem quanto à percepção dos impactos da TI nas variáveis estratégicas dos bancos.

Em princípio, ao fazer uma análise cross-country, não surpreende constatar diferenças de percepção, tendo em vista os resultados de diversos estudos comparativos realizados entre países, particularmente aqueles que focalizam diferenças culturais. Mesmo na indústria bancária, com grande experiência na utilização da TI, completamente imersa na globalização, as dife- renças emergem. Por outro lado, os resultados reforçam o que a literatura contemporânea de SI apregoa: as estratégias de TI devem alinhar-se com as estratégias globais da organização, sendo implementadas com o envolvimento de todos os executivos, e não apenas os de TI.

Quatro variáveis afetadas pela TI são os principais determinantes da competitividade na indústria bancária norte-americana: "internacionalização", "estrutura de custos e capacidade", "tomadores de recursos financeiros" e "fornecedores de recursos financeiros". Os executivos da indústria bancária norte-americana percebem os impactos da TI no processo de globalização das organizações onde atuam e destacam como relevante a possibilidade de ultrapassar barreiras geográficas, reduzir custos e aumentar capacidades, visando à satisfação do cliente. Em contraste, apenas uma variável surge como determinante da competitividade na indústria bancária brasileira: "estrutura de custos e capacidade". A percepção dos executivos brasileiros ainda está associada a uma visão limitada de back-office, restringindo o alcance da TI apenas à redução de custos e aumento de capacidades.

O estudo pode ser aprofundado, em futuras pesquisas, em várias direções. Uma delas, obviamente, é a extensão da comparação entre instituições bancárias de diferentes países, como a realizada por Lunardi (2001), que investigou a percepção dos executivos de bancos de diversos países latino-americanos. De outra parte, será interessante analisar se a percepção dos executivos quanto aos impactos da TI nas variáveis estratégicas difere segundo o porte da organização. Para isso, será necessário ampliar a amostra. Outra variável não investigada na pesquisa, merecedora de atenção, é a experiência no uso e aplicação da TI. Executivos de bancos inovadores no uso da TI perceberiam diferentemente seus impactos nas variáveis estratégicas organizacionais do que executivos de bancos seguidores (com estratégia seguidora) no uso da TI? Por outro lado, também parece ser interessante ampliar o estudo, replicando-o em diferentes tipos de organizações. Neste caso, haverá necessidade de adaptação do instrumento para os setores investigados. $\bigcirc$
NOTAS

Agradecemos as críticas e sugestões formuladas pelos avaliadores e editor do periódico.

A pesquisa recebeu apoio da University of Texas (EI Paso, EUA), CNPq, Capes e Fapergs.
1. A definição detalhada de cada variável do modelo não é apresentada devido a limitações de espaço neste artigo. Os autores colocam-se à disposição dos interessados para discussão.

2. Um teste $t$ de Student para amostras emparelhadas visando comparar a diferença de médias entre a variável "competitividade" e as demais foi realizado para cada subamostra, brasileira e norte-americana. Os resultados são significativos $(\alpha<0,05)$ para todas as comparações, exceto com a variável "requisitos de governos e países", para a subamostra brasileira, e com a variável "produtos e serviços", para a subamostra norte-americana. 


\section{REFERÊNCIAS BIBLIOGRÁFICAS}

ALBRIGHT, G. L., PARKER, M. Viva la revolución. Banking Strategies, v. 3, p. 33-45, July/Aug. 1998.

ARMSTRONG, C. P., SAMBAMURTHY, V. Information technology assimilation in firms: the influence of senior leadership and IT infrastructures. Information Systems Research, v. 10, n. 4, p. 304-327, Dec. 1999.

BAETS, W. R. J. Some empirical evidence on IS strategy alignment in banking. Information \& Management, v. 30, n. 4, p. 155-177, 1996.

BLANDEN, M. Size does matter. The Banker, v. 6, p. 30-34, June 1998

BROADBENT, M., WEILL, P. Improving business and information strategy alignment: learning from the banking industry. IBM Systems Journal, v. 12, n. 3. 1993.

CALLAHAN, C. V., NEMEC JR., J. The C.E.O'S information technology challenge: creating true value. Strategy \& Business, v. 1, n. 14, p. 78-89, 1999.

COMPANHIAS caminham para novas tecnologias. Gazeta Mercantil, São Paulo, 22 mar. 2001.

DEDRICK, J., KRAEMER, K. L. National technology policy and computer production in Asia-Pacific countries. The Information Society, v. 1, n. 11, p. 2958, 1995.

DEVLIN, J. F. Technology and innovation in retail banking distribution. International Journal of Bank Marketing, v. 13, n. 4, p. 19-25, 1995.

FEBRABAN. I Pesquisa nacional de tecnologia bancária - 1997. São Paulo : Ernst \& Young, 1997. Relatório de pesquisa.

GALBREATH, J. Web-based banking: creating a technology road map. Credit World, St. Louis, v. 22, n. 3, p. 24-28, May/June 1998.

HARTWICK, J. BARKI, H. Explaining the role of user participation in information system use. Management Science, v. 4, n. 40, p. 440-465, 1994.

HOFSTEDE, G. Cultures and organizations: software of the mind. London : McGraw-Hill, 1991.

JORDAN, J., KATZ, J. Banking in the age of information technology. Regional Review, Boston, $v$. 4, p. 24-30, 1999. Federal Reserve Bank of Boston.

JÖRESKOG, K. G., SÖRBOM, D. LISREL 8: structural equation modeling - user's reference guide. Chicago : Scientific Software, 1996.

KELLOWAY, E. K. Using LISREL for structural equation modeling: a researcher's guide. Los angeles: Sage, 1998.

KEMPIS, R. D. et al. Do IT smart: seven rules for superior information technology performance. New York : Free Press, 1999.

KHIAONARONG, T., LIEBENAU, J. Information technology in banking: a resource-based analysis. In: INFORMATION RESOURCES MANAGEMENT ASSOCIATION INTERNATIONAL CONFERENCE, 1999, Hershey, PA. Hershey, PA : IDEA Group, 1999. Managing information technology resources in organizations in the next millennium, p. 389-401.
LEITE, J. Imposto de Renda via Internet: inovando em serviços públicos através da tecnologia de informação. In: ENCONTRO ANUAL DA ASSOCIAÇÃO NACIONAL DOS PROGRAMAS DE PÓS-GRADUAÇÃO EM ADMINISTRAÇÃO, 22., 1998, Foz do Iguaçu. Anais... Foz do Iguaçu : Anpad, 1998. CD-ROM, Administração da Informação.

LUCAS JR., H. C. Information technology and the productivity paradox: assessing the value of investing in IT. New York : Oxford University Press, 1999.

LUNARDI, G. L. Os efeitos da tecnologia de informação (TI) nas variáveis estratégicas organizacionais da indústria bancária: estudo envolvendo países latinosamericanos. Dissertação (Mestrado em Administração) - PPGA/EA, Universidade Federal do Rio Grande do Sul, 2001.

MAÇADA, A. C. G., BECKER, J. L. Modelo para avaliar o impacto da tecnologia da informação (TI) nas variáveis estratégicas dos bancos brasileiros. In: ENCONTRO ANUAL DA ASSOCIAÇÃO NACIONAL DOS PROGRAMAS DE PÓS-GRADUAÇÃO EM ADMINISTRAÇÃO, 22., 1998, Foz do Iguaçu. Anais... Foz do Iguaçu : Anpad, 1998. CD-ROM, Administração da Informação.

MAÇADA, A. C. G., BECKER, J. L., MAHMOOD, M. Development and validation of an instrument to evaluate the impact of information technology investments: a cross country analysis. In: INFORMATION RESOURCES MANAGEMENT ASSOCIATION CONFERENCE (IRMA), 2000, Anchorage. Anchorage : IDEA Group, 2000.

MAHMOOD, M. A., SOON, S. K. A comprehensive model for measuring the potencial impact of information technology on organizational strategic variables. Decision Science,v. 22, n. 4, p. 869-897, 1991.

MILITELLO, K. Os campeões: ninguém bate os bancos em computação. Info Exame, São Paulo, p. 92-99, 1998.

MORISI, T. L. Comercial banking transformed by computer technology. Monthly Labor Review, v. 14, n. 6, p. 30-36, Aug. 1996.

MORRIS, S., MEED, J., SVENSEN, N. O gerente inteligente: como usar a tecnologia para alcançar o sucesso. São Paulo : Futura, 1998.

O'BRIEN, J. A. Management information systems: managing information technology in the internetworked enterprise. 4. ed. Boston : Irwin/ McGraw-Hill, 1999.

PALVIA, P. C. Developing a model of the global and strategic impact of information technology. Information and Management, n. 32, p. 229-244, 1997.

PEFFERS, K., DOS SANTOS, B. L. Performance effects of innovative IT applications over time. IEEE Transactions on Engineering Management, v. 43, n. 4, p. 381-392, Nov. 1996.

PEFFERS, K., TUUNAINEN, V. K. Expectations and impacts of a global information system: the case of a global bank from Hong Kong. Journal of Global Information Technology Management (JGITM), v. 1, n. 4, p. 17-37, 1998
PIRES, J. P. O perfil dos usuários de caixas automáticos em agências bancárias na cidade de Curitiba. Dissertação (Mestrado em Administração) - PPGA/SCSA, Universidade Federal do Paraná, 1996.

PORTER, M. E. Competitive strategy: techniques for analyzing industries and competitors. New York : Free Press, 1980.

PORTER, M., MILLAR, V. E. How information gives you competitive advantage. Harvard Business Review, Boston, v. 63, n. 4, p. 149-161, 1985.

ROCKART, J. F., EARL, M. J., ROSS, J. W. Eight imperatives for the new IT organization. Sloan Management Review, v. 38, n. 1, p. 43-55, Fall 1996.

ROTA, D. R. Strategic planning and future trends in information technology. In: ASSOCIATION INTERNATIONAL CONFERENCE, 1998, Boston. Boston : IDEA Group, 1998. Effective utilization and management of emerging information technologies, information resources management, v. 17, n. 20, p. 832-839.

RUIZ, F. J. M. Imagen y atracción de centros comerciales suburbanos. In: HAIR, J. F et al. Análisis multivariante. 5. ed. Madrid : Prentice Hall, 1999.

SAMBAMURTHY, V., ZMUD, R. W. Managing IT for success: the case of information technology. In: KOCHAN, T. A., USEEM, M. (Eds.). Transforming organizations. Oxford : Oxford University Press, 1992.

SAMBAMURTHY, V., ZMUD, R. W. IT management competency assessment: a tool for creating business value through IT. Morristown, NJ: Financial Executives Research Foundation, 1994

SCHIFRIN, M. The new enablers: chief information officers. Forbes, p. 138-143, June 1997.

SEGARS, A. H., GROVER, V. Re-examining perceived ease of use and usefulness: a confirmatory factor analysis. MIS Quarterly, v. 4, n. 17, p. 517-525, 1993.

SIJBRANDS, S., EPPINK, D. J. The internationalization of Dutch banks: a new beginning? Long Range Planning, v. 27, n. 4, p. 35-47, Aug. 1994.

STRASSMANN, P. A. The squandered computer: evaluating the business alignment of information technology. New Canaan, Connecticut: The Information Economics Press, 1997.

TAM, K. Y. The impact of information technology investments on firm performance and evaluation evidence from newly industrialized economies. Information Systems Research, v. 1, n. 9, p. 85-98, Mar. 1998.

WARNER, A. Foreign banks in Latin America: latin fever catches on. The Banker, p. 66-68, Jan. 1998.

ZANELA, A. C., FREITAS, H. M. R., BECKER, J. L. A influência da cultura e da experiência decisória sobre a percepção do processo decisório individual: um estudo comparativo inicial entre Brasil, França e Estados Unidos. In: ENCONTRO ANUAL DA ASSOCIAÇÃO NACIONAL DOS PROGRAMAS DE PÓS-GRADUAÇÃO EM ADMINISTRAÇÃO, 22., 1998 , Foz do Iguaçu. Anais... Foz do Iguaçu : Anpad, 1998. CD-ROM, Administração da Informação. 